\title{
PRODUCTS OF TWO BOREL MEASURES
}

\author{
BY
}

ROY A. JOHNSON

\begin{abstract}
Let $\mu$ and $\nu$ be finite Borel measures on Hausdorff spaces $X$ and $Y$, respectively, and suppose product measures $\mu \times_{1} \nu$ and $\mu \times_{2} \nu$ are defined on the Borel sets of $X \times Y$ by integrating vertical and horizontal cross-section measure, respectively. Sufficient conditions are given so that $\mu \times_{1} \nu=\mu \times_{2} \nu$ and so that the usual product measure $\mu \times \nu$ can be extended to a Borel measure on $X \times Y$ by means of completion. Examples are given to illustrate these ideas.
\end{abstract}

Throughout this paper $\mu$ and $\nu$ will be finite Borel measures on Hausdorff spaces $X$ and $Y$, respectively. That is, $\mu$ and $\nu$ will be countably additive, nonnegative real-valued measures on $\mathscr{B}(X)$ and $\mathscr{B}(Y)$, where $\mathscr{B}(X)$ and $\mathscr{B}(Y)$ are the Borel sets of $X$ and $Y$, respectively. Sometimes $\mu$ will be regular, which is to say that it is inner regular with respect to the compact sets. At times $\nu$ will be two-valued, which means that its range consists of the two values 0 and 1 . If $\nu$ can be expressed as the sum of a countable collection of multiples of two-valued Borel measures, then $\nu$ is purely atomic.

A (not necessarily regular) Borel measure $\mu$ will be called $\tau$-additive if $\mu\left(\cap F_{\alpha}\right)$ $=\inf \mu\left(F_{\alpha}\right)$ whenever $\left\{F_{\alpha}\right\}$ is a decreasing family of closed sets [4, p. 96]. Equivalently, $\mu$ is $\tau$-additive if $\mu\left(\cup V_{\alpha}\right)=\sup \mu\left(V_{\alpha}\right)$ for each increasingly directed family $\left\{V_{\alpha}\right\}$ of open sets. Of course, each regular Borel measure is $\tau$-additive.

A Borel rectangle is a set $A \times B$ such that $A$ and $B$ are Borel sets, and $\mathscr{B}(X) \times \Re(Y)$ denotes the $\sigma$-algebra generated by Borel rectangles. The notation $\mu \times \nu$ is reserved for the usual (not necessarily complete) product of $\mu$ and $\nu$, with $\mathscr{B}(X) \times \mathscr{B}(Y)$ for its domain.

If $M$ is a Borel set in $X \times Y$, let $M_{x}=\{y:(x, y) \in M\}$ for each $x \in X$ and let $M^{y}=\{x:(x, y) \in M\}$ for each $y \in Y$. If $M \in \mathscr{B}(X \times Y)$ and $\nu\left(M_{x}\right)$ is measurable as a function in $x$ (or $\mu$-almost everywhere equal to a measurable function), then we say that $\mu \times_{1} \nu(M)$ is defined and we let

$$
\mu \times_{1} \nu(M)=\int \nu\left(M_{x}\right) d \mu .
$$

If $\nu$ is $\tau$-additive or if $X$ is first countable, then Lemma 1.1 shows that $\nu\left(W_{x}\right)$ is lower semicontinuous for each open set $W$ in $X \times Y$. Hence, if $\nu$ is regular or if $X$ is first countable, then $\nu\left(M_{x}\right)$ is measurable as a function in $x$ for all $M \in$ $\mathscr{B}(X \times Y)$ so that $\mu \times_{1} \nu$ is a Borel measure on $X \times Y$. In the same manner, if

Received by the editors January 27, 1981.

1980 Mathematics Subject Classification. Primary 28A35; Secondary $28 \mathrm{C} 15$.

Key words and phrases. Borel measure, purely atomic measure, separable-regular Borel measure, completion of a measure. 
$M \in \mathscr{B}(X \times Y)$ and $\mu\left(M^{y}\right)$ is measurable (or $\nu$-almost everywhere equal to a measurable function), then $\mu \times_{2} \nu(M)$ is defined and

$$
\mu \times_{2} \nu(M)=\int \mu\left(M^{y}\right) d \nu .
$$

Notice that $\mu \times_{1} \nu(M)$ and $\mu \times_{2} \nu(M)$ are defined and equal to $\mu \times \nu(M)$ for all $M \in \mathscr{B}(X) \times \Re(Y)$.

The main purpose of this paper is to give conditions in $\$ 3$ such that each Borel set of $X \times Y$ can be sandwiched between two members of $\mathscr{B}(X) \times \mathscr{B}(Y)$ with the same $\mu \times \nu$-measure. (This happens, for example, if $\mu$ is inner regular with respect to the separable compact sets, if $X$ is first countable, and if $\nu$ is purely atomic.) In that case, $\mu \times_{1} \nu(M)$ and $\mu \times_{2} \nu(M)$ are defined and equal for all Borel sets $M$ in $X \times Y$. The existence and equality of $\mu \times_{1} \nu(M)$ and $\mu \times_{2} \nu(M)$ for all $M \in$ $\mathscr{B}(X \times Y)$ are of interest because they make possible the existence and equality of both iterated integrals for each nonnegative Borel function on $X \times Y$ [7, summary, p. 124].

I wish to thank D. H. Fremlin for helpful discussions concerning the ideas of this paper. In particular, Theorem 2.4 is due to him.

1. The $\mu \times_{1} \nu$-measure of an open set. In this section conditions are given under which each open set in $X \times Y$ contains a set in $\mathscr{B}(X) \times \mathscr{B}(Y)$ with the same $\mu \times_{1} \nu$-measure. For example, Theorem 1.2 shows that such containment occurs if $X$ is first countable and $\mu$ is regular.

LEMMA 1.1. Suppose $\nu$ is a Borel measure on $Y$, and suppose $W$ is an open set in $X \times Y$. Let $p \in X$, and let $\left\{U_{\alpha}: \alpha \in A\right\}$ be a base of open neighborhoods of $p$. If $\nu\left(W_{p}\right)>k$, then there exist $\alpha \in A$ and an open set $V$ such that $U_{\alpha} \times V \subseteq W$ and $\nu(V)>k$ if either of the following conditions holds:

(1) If $\kappa=\operatorname{card}(A)$, then the union of $\kappa$ Borel sets of $\nu$-measure zero has $\nu$-outer measure zero. (Of course, this condition is satisfied if $A$ is countable.)

(2) $\nu\left(\cup V_{\beta}\right)=\sup \nu\left(V_{\beta}\right)$ for each increasingly directed family $\left\{V_{\beta}\right\}$ of open sets in $Y$. That is, $\nu$ is $\tau$-additive.

Proof. Suppose $W$ is open in $X \times Y$ and that $\nu\left(W_{p}\right)>k$. For each $y \in W_{p}$, choose an open neighborhood $V(y)$ of $y$ such that $U_{\alpha} \times V(y) \subseteq W$ for some $\alpha \in A$. For each $\alpha \in A$, let $V_{\alpha}$ be the union of those $V(y)$ such that $U_{\alpha} \times V(y) \subseteq$ $W$. Then $V_{\beta} \subseteq V_{\gamma}$ whenever $U_{\beta} \supseteq U_{\gamma}$, and $W_{p}=\cup\left\{V_{\alpha}: \alpha \in A\right\}$. Hence, there is $\alpha \in A$ such that $\nu\left(V_{\alpha}\right)>k$. Since $U_{\alpha} \times V_{\alpha} \subseteq W$, we are done.

THEOREM 1.2. Suppose $\mu$ is a regular Borel measure on $X$ and that $\nu$ is a Borel measure on $Y$. Then each open set in $X \times Y$ contains an open set in $\mathscr{B}(X) \times \mathscr{B}(Y)$ with the same $\mu \times_{1} \nu$-measure if any one of the following conditions holds:

(1) $X$ is first countable.

(2) The character (minimum cardinality of a neighborhood base) of each point in $X$ is less than $\kappa$, and the union of fewer than $\kappa$ Borel sets of $\nu$-measure zero has $\nu$-outer measure zero.

(3) $\nu$ is $\tau$-additive. 
Proof. Let $W$ be open in $X \times Y$, and fix $n$. By Lusin's theorem, there exists a compact set $K$ in $X$ such that the restriction of the function $\nu\left(W_{x}\right)$ to $K$ is continuous and such that $\mu(X \backslash K)<1 / n$ [6, Exercise 55.3]. For each $p$ in $K$, we may choose by Lemma 1.1 open sets $U(p)$ and $V(p)$ such that

(i) $p \in U(p)$,

(ii) $\nu\left(W_{x}\right)<\nu\left(W_{p}\right)+1 / n$ for all $x \in U(p) \cap K$,

(iii) $\nu(V(p))>\nu\left(W_{p}\right)-1 / n$, and

(iv) $U(p) \times V(p) \subseteq W$.

Since $K$ is compact, there exists a finite collection of points $\left\{p_{i}\right\}$ in $K$ such that $K$ is covered by the corresponding collection of open sets $\left\{U\left(p_{i}\right)\right\}$. If

$$
W(n)=\bigcup\left\{U\left(p_{i}\right) \times V\left(p_{i}\right)\right\},
$$

then $W(n)$ is an open subset of $W$ such that $W(n) \in \mathscr{B}(X) \times \mathscr{B}(Y)$ and such that

$$
\mu \times_{1} \nu(W)<\mu \times{ }_{1} \nu(W(n))+(2 / n) \mu(X)+(1 / n) \nu(Y) .
$$

Then $W^{*}=U W(n)$ is the required open subset of $W$ with the same $\mu \times_{1} \nu$ measure as $W$.

The conclusion of Lemma 1.1 clearly holds if $\nu$ is regular. As a matter of fact, almost the same lemma can be proved if open sets of $Y$ are inner regular with respect to a class of sets having a certain type of property provided that the only sets in $X$ with that property are the closed sets. In order to make these ideas precise, let us say that a topological property $P$ is compact-like if whenever a set $A$ has property $P$, then so does

(i) the product of any compact set with $A$,

(ii) each closed subset of $A$, and

(iii) the image of $A$ under every continuous, open mapping. For example, countable compactness is a compact-like property. Now a set $A$ is called $\boldsymbol{\kappa}_{0}$ bounded (or strongly countably compact) if the closure in $A$ of each of its countable subsets is compact $\left(\left[5\right.\right.$, p. 201] or [12, pp. 763-764]). The property of $\boldsymbol{\aleph}_{0}$ boundedness is also a compact-like property.

Lemma 1.3. Suppose $\nu$ is a Borel measure on $Y$ such that each open set in $Y$ contains a set with a given compact-like property $P$ and with arbitrarily close $\nu$-outer measure. Suppose that every set in $X$ with property $P$ is closed. Suppose $W$ is an open set in $X \times Y$, that $K$ is a compact set in $X$, and that $p \in K$ is such that $\nu\left(W_{p}\right)>k$. Then there exist open sets $U$ and $V$ such that (i) $p \in U$, (ii) $\nu(V)>k$, and (iii) $(U \cap K) \times V \subseteq W$.

Proof. Since $W$ is open, we may choose a set $B$ contained in $W_{p}$ such that $B$ has property $P$ and such that the $\nu$-outer measure of $B$ is greater than $k$. Let

$$
\begin{aligned}
A & =\operatorname{Pr}_{X}((K \times B) \backslash W) \\
& =\{x \in X:(x, y) \in(K \times B) \backslash W \text { for some } y \in Y\} \\
& =K \backslash\left\{x \in K: B \subseteq W_{x}\right\} .
\end{aligned}
$$

Since $K \times B$ has property $P$, so do $(K \times B) \backslash W$ and $A$. Then $A$ is closed by hypothesis. Choose an open neighborhood $U$ of $p$ such that $\operatorname{closure}(U)$ is disjoint 
from $A$, and let $C=K \cap \operatorname{closure}(U)$. Clearly, $C \times B \subseteq W$. Now if $V=\{y \in Y$ : $C \times\{y\} \subseteq W\}$, it can be seen that $V$ is open [13, Theorem 5.12]. Moreover, $\nu(V)>k$ since $B \subseteq V$ and since the $\nu$-outer measure of $B$ exceeds $k$. Since $C \times V \subseteq W$, we see that $(U \cap K) \times V \subseteq W$ and we are done.

A set $A$ in a topological space is called sequentially closed if every convergent sequence in $A$ has its limit in $A$. A space is called sequential if every sequentially closed set is closed [2, pp. 108-109]. Every countably compact set in a sequentially closed space is closed. A topological space is called countably tight if for every subset $A$, each limit point of $A$ is a limit point of some countable subset of $A$. Notice that each $\aleph_{0}$-bounded set in a countably tight space is closed. Moreover, each first countable space is sequential and each sequential space is countably tight.

In (1) of the next theorem and in the remarks following Theorem 2.5 we shall have occasion to use the condition that each compact set $K$ in $X$ contains a point having a countable base of neighborhoods in $K$. This condition is equivalent to saying that the set of points in $K$ having a countable base of neighborhoods in $K$ forms a dense set in the compact set $K$. This condition is satisfied if $\operatorname{card}(X)<2^{\boldsymbol{\kappa}_{1}}$ [10, Theorem 2.24].

THEOREM 1.4. Suppose $\mu$ is a regular Borel measure on $X$ and that $\nu$ is a Borel measure on $Y$. Then each open set in $X \times Y$ contains a (not necessarily open) set in $\Re(X) \times \Re(Y)$ with the same $\mu \times_{1} \nu$-measure if any one of the following conditions holds:

(1) Each compact set $K$ in $X$ contains a point with a countable base of neighborhoods in $K$, and $\mu \times_{1} \nu(M)$ is defined for each Borel set $M$ in $X \times Y$.

(2) Each compact set $K$ in $X$ contains a point with character (minimum cardinality of a neighborhood base) less than $\kappa$, the union of fewer than $\kappa$ Borel sets in $Y$ of $\nu$-measure zero has $\nu$-outer measure zero, and $\mu \times_{1} \nu(M)$ is defined for each Borel set $M$ in $X \times Y$.

(3) $X$ is countably tight, and each open set in $Y$ contains an $\aleph_{0}$-bounded set with arbitrarily close $\nu$-outer measure.

(4) $X$ is sequential, and each open set in $Y$ contains a countably compact set with arbitrarily close $\nu$-outer measure.

Proof. Suppose $W$ is open in $X \times Y$. Let $n \geqslant 1$. Let $\left\{A_{i}\right\}$ be a maximal disjoint family of Borel sets in $X$ such that $\mu\left(A_{i}\right)>0$ for each $A_{i}$ and such that for each $A_{i}$ there exists a corresponding Borel set $B_{i}$ in $Y$ such that $A_{i} \times B_{i} \subseteq W$ and such that $\nu\left(W_{x} \backslash B_{i}\right)<2 / n$ for each $x \in A_{i}$. Since $\mu(X)$ is finite, the family $\left\{A_{i}\right\}$ is necessarily countable. It suffices to show that $\mu\left(X \backslash \cup A_{i}\right)=0$ since in that case $W(n)=\cup A_{i} \times B_{i}$ will be a subset of $W$ such that $W(n) \in \mathscr{B}(X) \times \mathscr{B}(Y)$ and such that

$$
\mu \times_{1} \nu(W) \leqslant \mu \times_{1} \nu(W(n))+(2 / n) \mu(X) .
$$

Suppose otherwise that $\mu\left(X \backslash \cup A_{i}\right)>0$. Since $\mu$ is regular, there exists by Lusin's theorem a compact set $K$ contained in $X \backslash \cup A_{i}$ such that $\mu(K)>0$ and such that the restriction of the function $\nu\left(W_{x}\right)$ to $K$ is continuous [6, Exercise 55.3]. 
We may assume that each nonempty relatively open subset of $K$ has positive $\mu$-measure. Assuming condition (1) [resp., condition (2)], choose a point $p$ in $K$ with a countable base of neighborhoods in $K$ [resp., with character less than $\kappa$ in $K$ ]. Assuming conditions (3) or (4), let $p$ be any point in $K$. By Lemma 1.1 or Lemma 1.3, we may find an open set $U$ in $X$ and an open set $V$ in $Y$ such that (i) $p \in U$, (ii) $\nu(V)>\nu\left(W_{p}\right)-1 / n$, and (iii) $(U \cap K) \times V \subseteq W$. Because $\nu\left(W_{x}\right)$ is continuous on $K$, we may assume that $\nu\left(W_{x}\right)<\nu\left(W_{p}\right)+1 / n$ for all $x \in U \cap K$ so that $\nu\left(W_{x} \backslash V\right)<2 / n$ for each $x \in U \cap K$. Since $U \cap K$ is a nonempty open set in $K$, we see that $\mu(U \cap K)>0$. Hence, $U \cap K$ violates the maximality of the family $\left\{A_{i}\right\}$, so that $\mu\left(K \backslash \cup A_{i}\right)=0$ as was to be shown.

2. The $\mu \times_{1} \nu$-measure of a closed set. This section is "dual" to the preceding section in that conditions are given under which a closed set in $X \times Y$ contains a set in $\mathscr{B}(X) \times \mathscr{B}(Y)$ with the same $\mu \times_{1} \nu$-measure. Whether the appropriate condition on $\mu$ holds if $\mu$ is regular and $X$ is first countable depends on the set theory that is assumed.

Suppose $\mu$ is a (not necessarily regular) Borel measure on $X$. Let us say that $\mu$ is separable-regular if each Borel set $E$ in $X$ contains a countable subset $C$ such that $\mu(E \backslash$ closure $(C))=0$. In other words, $\mu$ is separable-regular if it inner regular with respect to the class of separable Borel sets. If $X$ is hereditarily separable, then every Borel measure on $X$ is separable-regular. Similarly, every Borel measure on a metric space whose cardinality has measure zero is separable-regular [15, Theorem III]. Finally, each regular Borel measure on a totally ordered space is separableregular.

Notice that the following theorem does not require regularity of any of the measures. The condition that $\nu$ be purely atomic is restrictive, and it may be possible to weaken or even drop that requirement.

THeOREM 2.1. Suppose $\nu$ is a purely atomic Borel measure on $Y$, that $\mu$ is separable-regular, and that $\mu \times_{1} \nu(M)$ is defined for all Borel sets $M$ in $X \times Y$. (These conditions on $\mu$ and $\mu \times_{1} \nu$ are satisfied, for example, if $X$ is hereditarily separable.) Then each closed set in $X \times Y$ contains a closed set in $\mathscr{B}(X) \times \mathscr{B}(Y)$ with arbitrarily close $\mu \times_{1} \nu$-measure. Hence, each closed set in $X \times Y$ contains a set in $\mathscr{B}(X) \times \mathscr{B}(Y)$ with the same $\mu \times_{1} \nu$-measure. In particular, $\mu \times_{1} \nu(F) \leqslant$ $\mu \times_{2} \nu(F)$ if $F$ is a closed set in $X \times Y$ such that $\mu \times_{2} \nu(F)$ is defined.

Proof. Suppose $\mu$ is separable-regular and that $\mu \times_{1} \nu(M)$ is defined for all Borel sets $M$ in $X \times Y$. We may assume without loss of generality that $\nu$ is two-valued. Let $F$ be closed in $X \times Y$, and let $E=\left\{x \in X: \nu\left(F_{x}\right)=1\right\}$. Since $\mu$ is separable-regular, there exists a countable set $C$ in $E$ such that the $\mu$-outer measure of $E \backslash$ closure $(C)$ is zero. Let $B=\cap\left\{F_{x}: x \in C\right\}$. Clearly, $B$ is closed and $\nu(B)=1$. If $A=$ closure $(C)$ and $x \in A$, it follows that $B \subseteq F_{x}$ since $F$ is closed. Hence, $A \times B \subseteq F$ and $\mu \times_{1} \nu(A \times B)=\mu(A)=\mu \times_{1} \nu(F)$.

A variation of Theorem 2.1 holds if $\nu$ satisfies a stronger condition than countable additivity. That is, suppose $\nu$ is a purely atomic Borel measure such that 
the union of fewer than $\kappa$ Borel sets of $\nu$-measure zero has $\nu$-outer measure zero. Suppose each Borel set $E$ in $X$ contains a subset $C$ such that $\operatorname{card}(C)<\kappa$ and such that $\mu(E \backslash$ closure $(C))=0$. In other words, suppose $\mu$ is inner regular with respect to the class of Borel sets in $X$ with density less than $\kappa$. If $F$ is a closed set in $X \times Y$ such that $\mu \times_{1} \nu(F)$ is defined, then $F$ contains a closed set in $\mathscr{B}(X) \times \mathscr{B}(Y)$ with arbitrarily close $\mu \times_{1} \nu$-measure. The proof of the preceding assertion is analogous to that of Theorem 2.1 .

In view of the next theorem, separable-regularity of $\mu$ cannot be replaced by regularity of $\mu$ in Theorem 2.1. Under the Continuum Hypothesis the hypotheses of Theorem 2.2 can be satisfied with $\kappa=\omega_{1}$ by a regular Borel measure $\mu$ on a first countable, hereditarily Lindelöf space [14]. A point $x \in X$ will be said to have tightness less than $\kappa$ if whenever $x \in \operatorname{closure}(A)$, there exists a subset $C$ of $A$ with $\operatorname{card}(C)<\kappa$ such that $x \in \operatorname{closure}(C)$.

THEOREM 2.2. Let $\kappa$ be a regular cardinal, and suppose each point of $X$ has tightness less than $\kappa$. Suppose $\mu(\operatorname{closure}(C))=0$ whenever card $(C)<\kappa$, and suppose $\mu($ closure $(C))>0$ for some $C$ with cardinality $\kappa$. Let $Y$ be the set of ordinals less than or equal to $\kappa$, and let $Y$ have the order topology. Then there exist a two-valued Borel measure $\nu$ on $Y$ and a closed set $F$ in $X \times Y$ such that $\mu \times_{1} \nu(F)>0$ and $\mu \times_{2} \nu(F)=0$.

Proof. Let $Y_{0}=Y \backslash\{\kappa\}$, and let $\nu$ be the two-valued Borel measure on $Y$ such that $\nu(D)=1$ if and only if $D$ contains a closed, unbounded subset of $Y_{0}$ (cf. [6, Exercise 52.10]). Since $\kappa$ is a regular cardinal, the intersection of fewer than $\kappa$ closed, unbounded sets in $Y_{0}$ is itself a closed, unbounded set in $Y_{0}$. It follows that $\nu$ is a measure; indeed, the intersection of fewer than $\kappa$ Borel sets of $\nu$-measure 1 contains a Borel set with measure 1.

Let $C$ be a subset of $X$ of cardinality $\kappa$ such that $\mu(\operatorname{closure}(C))>0$, and let $g$ : $C \rightarrow Y_{0}$ be a one-to-one correspondence between $C$ and $Y_{0}$. Let

$$
H=\{(x, y) \in C \times Y: g(x) \leqslant y\},
$$

and let $F$ be the closure of $H$ in $X \times Y$. If $x \in C$, then $F_{x}$ contains $\{y \in Y$ : $g(x) \leqslant y$ \}. Thus $\nu\left(F_{x}\right)=1$ for all $x$ in $C$. Since each point of $X$ has tightness less than $\kappa$, we then have $\nu\left(F_{x}\right)=1$ for all $x$ in closure $(C)$. Therefore, $\mu \times_{1} \nu(F) \geqslant$ $\mu(\operatorname{closure}(C))>0$.

If $y \in Y$, then $H^{y}=\{x: g(x) \leqslant y\}$. Hence, $\mu$ (closure $\left.\left(H^{y}\right)\right)=0$ if $y \in Y_{0}$. Since $H^{z} \subseteq H^{y}$ whenever $z \leqslant y$, it follows that $F^{y}=\operatorname{closure}\left(H^{y}\right)$ for all $y$ in $Y$. Hence, $\mu\left(F^{y}\right)=0$ for all $y \in Y_{0}$, so that $\mu \times_{2} \nu(F)$ is defined and equal to zero.

In the preceding theorem, $\mu \times_{1} \nu(M)$ is defined for all Borel sets $M$ in $X \times Y$. If $\mu$ is regular, then $\mu \times_{2} \nu(M)$ is defined for all Borel sets $M$ in $X \times Y$ also.

Corollary 2.3. Suppose $X$ is countably tight and that $\operatorname{card}(X)<\boldsymbol{\aleph}_{\omega}$. If $\mu$ is not separable-regular, then there exist a two-valued Borel measure $\nu$ on a compact space $Y$ and $a$ closed set $F$ in $X \times Y$ such that $\mu \times_{1} \nu(F)>0$ and $\mu \times_{2} \nu(F)=0$. 
TheOREM 2.4 (Fremin). Assume Martin's Axiom. Then there is a compact space $X$ such that each of its points has character (minimum cardinality of a neighborhood base) less than $2^{\omega}$ and a nonzero regular Borel measure $\mu$ on $X$ such that $\mu(\operatorname{closure}(C))=0$ for every subset $C$ in $X$ with cardinality less than $2^{\omega}$.

Proof. Let $Z$ be the space of all functions from $\omega$ into a discrete space of two elements, and let $Z$ have the product topology. Let $\lambda$ be Haar measure (the usual product measure) on $Z$, and label the points of $Z$ as $\left\{z_{\alpha}: \alpha<2^{\omega}\right\}$. For each $\alpha<2^{\omega}$, let $Z_{\alpha}=\left\{z_{\beta}: \beta<\alpha\right\}$. In view of Martin's Axiom, each $Z_{\alpha}$ has $\lambda$-outer measure zero [16, §4.1]. For each $\alpha<2^{\omega}$ and each $n$, we may thus choose an open set $G_{\alpha, n}$ in $Z$ such that $Z_{\alpha} \subseteq G_{\alpha, n}$ and $\lambda\left(G_{\alpha, n}\right)<1 / n$.

Let $\mathcal{E}$ be the family consisting of the sets $\left\{G_{\alpha, n}\right\}$, their complements in $Z$, and the clopen sets in $Z$. Let $\mathcal{Q}$ be the smallest algebra of subsets of $Z$ containing $\mathcal{E}$, and notice that each member of $Q$ can be written as a finite union of finite intersections of members of $\mathcal{E}$.

Let $X$ be the Stone space of $\mathbb{Q}\left[13\right.$, Exercise 5.S]. For each $A \in \mathbb{Q}$, write $A^{*}$ for the corresponding clopen set in $X$. The restriction of $\lambda$ to $Q$ induces a regular Borel measure $\mu$ on $X$ such that $\mu\left(A^{*}\right)=\lambda(A)$ for each $A \in \mathbb{Q}$. Let $f$ be the continuous map from $X$ onto $Z$ given by $f(x)=y$, where

$$
\{y\}=\bigcap\left\{U: U \text { is clopen in } Z \text { and } x \in U^{*}\right\} .
$$

If $f(x) \in G_{\alpha, n}$, we observe that $x \in G_{\alpha, n}^{*}$; indeed, there exists a clopen set $U$ in $Z$ such that $x \in U^{*} \subseteq G_{\alpha, n}^{*}$.

We check that each point of $X$ has character less than $2^{\omega}$. Suppose $x \in X$. Then a base of neighborhoods of $x$ is $\left\{A^{*}: A \in \mathbb{Q}\right.$ and $\left.x \in A^{*}\right\}$, so that a subbase of neighborhoods of $x$ is $\left\{E^{*}: E \in \mathcal{E}\right.$ and $\left.x \in E^{*}\right\}$. We know that $f(x)=z_{\beta}$ for some $\beta<2^{\omega}$, so that $f(x) \in G_{\alpha, n}$ for all $\alpha$ satisfying $\beta<\alpha<2^{\omega}$ and for all $n$. Hence, $x$ has a subbase of neighborhoods with cardinality less than $2^{\omega}$, namely

$$
\begin{gathered}
\left\{U^{*}: U \text { is a clopen set in } Z \text { and } x \in U^{*}\right\} \cup\left\{G_{\alpha, n}^{*}: \alpha \leqslant \beta \text { and } x \in G_{\alpha, n}^{*}\right\} \\
\cup\left\{X \backslash G_{\alpha, n}^{*}: \alpha \leqslant \beta \text { and } x \in X \backslash G_{\alpha, n}^{*}\right\} .
\end{gathered}
$$

Hence, $x$ has a base of neighborhoods with cardinality less than $2^{\omega}$ (cf. [13, p. 50]).

We now show that if $C$ is a subset of $X$ with cardinality less than $2^{\omega}$, then $\mu(\operatorname{closure}(C))=0$. Since $f[C]$ has cardinality less than $2^{\omega}$, there exists $\alpha<2^{\omega}$ such that $f[C] \subseteq Z_{\alpha}$. Thus for each positive integer $n$, we have $f[C] \subseteq G_{\alpha, n}$. Hence, $C \subseteq G_{\alpha, n}^{*}$ so that closure $(C)$ is a subset of $G_{\alpha, n}^{*}$. Then since $\mu\left(G_{\alpha, n}^{*}\right)=\lambda\left(G_{\alpha, n}\right)<1 / n$ for all $n$, we have $\mu(\operatorname{closure}(C))=0$.

It is consistent with the usual axioms of set theory (ZFC) to suppose that there exists a regular Borel measure $\mu$ on a first countable, compact space such that $\mu$ fails to be separable-regular. On the other hand, Theorem 2.5 shows that it is consistent to suppose that each regular Borel measure on a first countable space is separable-regular.

THEOREM 2.5. Assume Martin's Axiom and the negation of the Continuum Hypothesis. If $X$ is first countable and if $\mu$ is regular, then $\mu$ is separable-regular. 
Proof. Since $\mu$ is regular, it suffices to show that each compact set contains a separable compact set with the same $\mu$-measure. Suppose that $K$ is compact. Without loss of generality we may suppose that $K$ contains no proper closed set with the same $\mu$-measure. Then $\mu(U \cap K)>0$ whenever $U$ is an open set in $X$ and $U \cap K$ is nonempty. Hence, every disjoint collection of nonempty open sets in the subspace $K$ is countable. This means that $K$ is a first countable, compact space satisfying the countable chain condition (having countable cellularity). Assuming Martin's Axiom and the negation of the Continuum Hypothesis, such a space $K$ is separable and we are done [10, Theorem 5.6].

The hypothesis that $X$ be first countable in Theorem 2.5 can be replaced by the hypotheses that $X$ has countable tightness and that each compact set $K$ in $X$ contains a point having a countable base of neighborhoods in $K$. The essential details are found in [10, Theorems 2.25, 2.26, 5.6 and corollary of 5.4].

3. Borel sets and the completion of $\mu \times \nu$. Results from the first two sections can now be combined to give conditions under which every Borel set in $X \times Y$ can be sandwiched between two sets of $\mathscr{B}(X) \times \mathscr{B}(Y)$ with the same $\mu \times \nu$-measure. When that happens, the product measures $\mu \times_{1} \nu$ and $\mu \times_{2} \nu$ will be defined and equal for all Borel sets in $X \times Y$.

It is easy to check that the following statements are equivalent.

I. Each Borel set in $X \times Y$ is $\mu \times \nu$-measurable.

II. Each Borel set in $X \times Y$ lies in the domain of completion of $\mu \times \nu$.

III. $\mu \times_{1} \nu(M)$ is defined for all $M \in \mathscr{B}(X \times Y)$, and $\mu \times_{1} \nu$ and $\mu \times \nu$ have the same completion.

IV. $\mu \times_{1} \nu(M)$ is defined for all $M \in \mathscr{B}(X \times Y)$, and each open or closed set in $X \times Y$ contains a set in $\mathscr{B}(X) \times \mathscr{B}(Y)$ with the same $\mu \times_{1} \nu$-measure.

In view of statement IV, we may combine Theorems 1.2, 1.4, and 2.1 as follows:

THEOREM 3.1. Suppose $\mu$ is a regular, separable-regular Borel measure on $X$ and that $\nu$ is a purely atomic Borel measure on $Y$. Then the domain of completion of $\mu \times \nu$ includes the Borel sets of $X \times Y$ if any one of the following conditions holds:

(1) $X$ is first countable.

(2) $X$ is sequential, and each open set in $Y$ contains a countably compact set with arbitrarily close $\nu$-outer measure.

(3) $X$ is countably tight, and each open set in $Y$ contains an $\aleph_{0}$-bounded set with arbitrarily close $\nu$-outer measure.

(4) $\mu \times_{1} \nu(M)$ is defined for all $M \in \Re(X \times Y)$, and each compact set $K$ in $X$ contains a point with a countable base of neighborhoods in $K$.

(5) $\nu$ is regular.

Alternate statements of Theorem 3.1 are possible if $\nu$ satisfies a stronger condition than countable additivity. For example, if $\nu$ is a purely atomic Borel measure on $Y$ such that the union of fewer than $\kappa$ sets of $\nu$-measure zero has $\nu$-outer measure zero, if each point of $X$ has character less than $\kappa$, and if $\mu$ is a regular Borel measure such that each Borel set in $X$ contains a Borel set with the 
same $\mu$-measure and with density (cardinality of a dense subset) less than $\kappa$, then the Borel sets of $X \times Y$ lie in the domain of completion of $\mu \times \nu$.

Assuming Martin's Axiom and the negation of the Continuum Hypothesis, we know by Theorem 2.5 that regular Borel measures on first countable spaces are separable-regular. We thus have the following corollary to Theorem 3.1.

Corollary 3.2. Assume Martin's Axiom and the negation of the Continuum Hypothesis. If $\mu$ is a regular Borel measure on the first countable space $X$ and if $\nu$ is a purely atomic Borel measure on $Y$, then the domain of completion of $\mu \times \nu$ includes the Borel sets of $X \times Y$.

In view of Theorem 2.2, the conclusion of Corollary 3.2 can fail in the presence of the Continuum Hypothesis. On the other hand, the next theorem shows that each Borel set of $X \times Y$ can be $\mu \times \nu$-measurable even if $\mu$ and $\nu$ both fail to be regular.

TheOREM 3.3. Suppose $\mu$ is a Borel measure on $X$ and that $X$ has cardinality $\kappa$. Suppose $\nu$ is a purely atomic Borel measure on $Y$ such that the union of $\kappa$ sets of $\nu$-measure zero has $\nu$-outer measure zero. Then the Borel sets of $X \times Y$ lie in the domain of completion of $\mu \times \nu$.

Proof. We may assume without loss of generality that $\nu$ is two-valued. We show first that $\mu \times_{1} \nu(M)$ is defined for each Borel set $M$ in $X \times Y$. If $F$ is closed in $X \times Y$, let $A=\left\{x: \nu\left(F_{x}\right)=1\right\}$. If $B=\cap\left\{F_{x}: x \in A\right\}$, then $B$ is a closed set and $\nu(B)=1$. If $p$ is a limit point of $A$, then $B \subseteq F_{p}$ so that $p \in A$. Hence, $A$ is closed. It follows that $\left\{x: \nu\left(M_{x}\right)=1\right\}$ is a Borel set in $X$ for each $M \in \mathscr{B}(X \times Y)$.

Now suppose $M$ is a Borel set in $X \times Y$, and let $A=\left\{x: \nu\left(M_{x}\right)=1\right\}$. If $D=\cap\left\{M_{x}: x \in A\right\}$, then $D$ contains a Borel set $B$ such that $\nu(B)=1$. Since $A \times B \subseteq M$ and since $\mu \times_{1} \nu(M)=\mu \times_{1} \nu(A \times B)$, we see that each Borel set of $X \times Y$ lies in the domain of completion of $\mu \times \nu$.

4. The $\mu \times_{2} \nu$-measure of a closed set. The main result of this section is Corollary 4.5, which gives a sufficient condition for each closed set in $X \times Y$ to contain a set in $\mathscr{B}(X) \times \mathscr{B}(Y)$ with the same $\mu \times_{2} \nu$-measure, where $\mu$ is regular and $\nu$ is purely atomic. We begin with a lemma that will be used in the proof of Theorem 4.4.

Lemma 4.1 (CF. [7, TheOREM 4.1]). Suppose $F$ is closed in $X \times Y$, and suppose $q \in Y$. If $F^{q} \subseteq U$ and $X \backslash U$ is compact, then there exists a neighborhood $V$ of $q$ such that $F^{y} \subseteq U$ for all $y \in V$.

Proof. Since $(X \backslash U) \times\{q\}$ is a compact subset of the open set $(X \times Y) \backslash F$, there exists an open rectangle $G \times V$ containing $(X \backslash U) \times\{q\}$ such that $G \times V$ $\subseteq(X \times Y) \backslash F\left[13\right.$, Theorem 5.12]. Then $F^{y} \subseteq X \backslash G \subseteq U$ for all $y \in V$.

It will be convenient to say that the product measure $\mu \times_{2} \nu$ is well behaved with respect to $\mathscr{B}(X) \times \mathscr{B}(Y)$ or simply well behaved if $\mu \times_{2} \nu$ is defined for all $M \in \mathscr{B}(X \times Y)$ and if for each $M$ in $\mathscr{B}(X \times Y)$, there exists a set $N$ in $\mathscr{B}(X) \times \mathscr{B}(Y)$ such that $\mu \times_{2} \nu(M \triangle N)=0$. Equivalently, $\mu \times_{2} \nu$ is well behaved if for each $M \in \mathscr{B}(X \times Y)$, there exists a set $N$ in $\mathscr{B}(X) \times \mathscr{B}(Y)$ such that 
$\mu\left(M^{y} \triangle N^{y}\right)=0$ for $\nu$-almost all $y$ in $Y$. If each Borel set of $X$ lies in the domain of completion of $\mu \times \nu$ (see, for example, §3), then clearly $\mu \times_{2} \nu$ is well behaved. We shall not have occasion to use the corresponding concept of $\mu \times_{1} \nu^{\prime}$ being well behaved except to point out that $\mu \times_{2} \nu$ can be well behaved even if $\mu \times_{1} \nu$ is not well behaved.

Theorem 4.2. Suppose $\mu \times_{2} \nu(M)$ is defined for all Borel sets $M$ in $X \times Y$. Then the following are equivalent:

(1) $\mu \times_{2} \nu$ is well behaved with respect to $\Re(X) \times \mathscr{B}(Y)$.

(2) For each Borel set $M$ in $X \times Y$ and each $\varepsilon>0$, there exists $N \in \mathscr{B}(X) \times$ $\mathscr{B}(Y)$ such that $\mu \times_{2} \nu(M \triangle N)<\varepsilon$.

(2') For each Borel set $M$ in $X \times Y$ and each $\varepsilon>0$, there exists a finite collection of Borel rectangles $\left\{A_{i} \times B_{i}\right\}$ such that $\mu \times_{2} \nu\left(M \triangle \cup\left(A_{i} \times B_{i}\right)\right)<\varepsilon$.

(3) For each closed set $F$ in $X \times Y$ and each $\varepsilon>0$, there exists a set $N$ in $\mathscr{B}(X) \times \mathscr{B}(Y)$ such that $\mu \times_{2} \nu(F \triangle N)<\varepsilon$.

(3') For each closed set $F$ in $X \times Y$ and each $\varepsilon>0$, there exists a finite collection of Borel rectangles $\left\{A_{i} \times B_{i}\right\}$ such that $\mu \times_{2} \nu\left(F \triangle \cup\left(A_{i} \times B_{i}\right)\right)<\varepsilon$.

(4) For each closed set $F$ in $X \times Y$, there exists a set $N$ in $\mathscr{B}(X) \times \mathscr{B}(Y)$ such that $\mu \times_{2} \nu(F \triangle N)=0$.

Proof. We first prove that (3) implies (4). Suppose $F$ is a closed set in $X \times Y$. We find for each $n$ a set $N_{n}$ in $\mathscr{B}(X) \times \mathscr{B}(Y)$ such that $\mu \times_{2} \nu\left(F \triangle N_{n}\right)<1 / n$. Then $\left\{N_{n}\right\}$ is fundamental in measure with respect to $\mu \times_{2} \nu$ (and hence $\mu \times \nu$ ) in the sense that the corresponding sequence of characteristic functions is fundamental in measure [1, p. 60]. Hence, there is a set $N$ in $\mathscr{B}(X) \times \mathscr{B}(Y)$ such that the sequence $\left\{N_{n}\right\}$ converges to $N$ in $\mu \times \nu$-measure [1, Theorem 21.4]. Since $\left\{N_{n}\right\}$ converges to both $F$ and $N$ in $\mu \times_{2} \nu$-measure, we see that $F$ and $N$ are equal almost everywhere with respect to $\mu \times_{2} \nu$ [1, Theorem 20.3]. In other words, $\mu \times_{2} \nu(F \triangle N)=0$.

In order to prove that (4) implies (1), let $\delta$ be the class of all Borel sets $M$ in $X \times Y$ such that $\mu \times_{2} \nu(M \triangle N)=0$ for some $N \in \mathscr{B}(X) \times \mathscr{B}(Y)$. Then $\delta$ is a $\sigma$-algebra. Assuming (4), $\mathcal{S}$ contains the closed sets and hence the Borel sets of $X \times Y$. The remaining implications are obvious.

THEOREM 4.3. Suppose $\mu$ is a $\tau$-additive Borel measure which is inner regular with respect to the closed sets. (This is the case if $\mu$ is a regular Borel measure.) Suppose $\nu$ is a two-valued Borel measure. Then $\mu \times_{2} \nu$ is well behaved with respect to $\mathscr{B}(X) \times$ $\mathscr{B}(Y)$ if and only if each closed set in $X \times Y$ contains a closed rectangle with the same $\mu \times_{2} \nu$-measure.

Proof. Suppose $\mu \times_{2} \nu$ is well behaved, and suppose $F$ is a closed set in $X \times Y$. If $\mu \times_{2} \nu(F)=0$, then the empty set will serve as the required rectangle. Hence, we may assume that $\mu \times_{2} \nu(F)>0$. By Theorem 4.2(4), there exists $N \in \mathscr{B}(X) \times$ $\mathscr{B}(Y)$ such that $\mu \times_{2} \nu(F \triangle N)=0$. Now if $\mathcal{S}$ is the class of all sets in $\mathscr{B}(X) \times$ $\mathscr{B}(Y)$ which contain a Borel rectangle with the same $\mu \times_{2} \nu$-measure, then $\delta$ is a $\sigma$-algebra containing the class of Borel rectangles. Thus $N$ contains a Borel 
rectangle $A \times B$ with the same $\mu \times_{2} \nu$-measure, so that $\mu \times_{2} \nu(F \triangle(A \times B))=0$. Necessarily, $\mu\left(F^{y} \triangle A\right)=0$ for almost all $y$ in $B$. Hence, if $D=\left\{y: \mu\left(F^{y} \triangle A\right)=\right.$ $0\}$, then $\nu(D)=1$.

Fix $q \in D$, and notice that $\mu\left(F^{q} \triangle F^{y}\right) \leqslant \mu\left(F^{q} \triangle A\right)+\mu\left(A \triangle F^{y}\right)=0$ for all $y \in D$. Choose a sequence of closed sets $\{C(n)\}$ contained in $F^{q}$ such that $\mu(C(n))>\mu\left(F^{q}\right)-1 / n$. Since $\mu$ is $\tau$-additive, we may arrange to have $\mu(C(n) \cap U)>0$ whenever $U$ is an open set such that $C(n) \cap U$ is nonempty. If $y \in D$, then $\mu\left(C(n) \backslash F^{y}\right) \leqslant \mu\left(F^{q} \backslash F^{y}\right)=0$ so that $C(n) \subseteq F^{y}$ for all $n$. Hence, if $C=\cup C(n)$, then $C \times D \subseteq F$. We observe that $C \times D$ has the same $\mu \times{ }_{2} \nu$ measure as $F$ since

$$
\mu \times_{2} \nu(C \times D) \geqslant \mu \times_{2} \nu(C(n) \times D)>\mu \times_{2} \nu(F)-1 / n
$$

for each $n$. Since $F$ is closed, it contains closure $(C \times D)$. The reverse implication is clear.

In most cases, if $\mu$ is regular and $\nu$ is two-valued, then our next theorem will show that $\mu \times_{2} \nu$ is well behaved. (Assuming the existence of a measurable cardinal, an exception is given in Example 5.4.) Recall that a point $q$ is an accumulation point of a set $E$ if each neighborhood of $q$ contains infinitely many members of $E$. We shall say that a Borel set $V$ in $Y$ is $\nu$-concentrated if there exists a subset $D$ of $V$ such that

(i) the $\nu$-inner measure of $V \backslash D$ is zero (or equivalently, $D$ meets each Borel set $B$ such that $\nu(V \cap B)>0)$, and

(ii) if there exist $\kappa$ Borel sets in $V$ of $\nu$-measure zero whose union has positive $\nu$-outer measure and if $E$ is a subset of $D$ with cardinality $\kappa$, then $E$ has an accumulation point in $V$.

TheOREM 4.4. Suppose $\mu$ is a regular Borel measure, and suppose $\nu$ is a two-valued Borel measure such that every open set $V$ in $Y$ is $\nu$-concentrated. Then $\mu \times_{2} \nu$ is well behaved with respect to $\mathscr{B}(X) \times \mathscr{B}(Y)$. Hence, each closed set in $X \times Y$ contains a closed rectangle with the same $\mu \times_{2} \nu$-measure in this case.

Proof. Suppose $\mu \times_{2} \nu$ is not well behaved. Then by Theorem 4.2(3) there exists a closed set $F$ in $X \times Y$ and $\varepsilon>0$ such that $\mu \times{ }_{2} \nu(F \triangle N)>\varepsilon$ for each set $N \in \mathscr{B}(X) \times \mathscr{B}(Y)$. Let

$$
B=\left\{y \in Y: \mu\left(F^{y}\right)=\mu \times_{2} \nu(F)\right\},
$$

and let

$$
V=\left\{y \in Y: \mu\left(F^{y}\right)<\mu \times_{2} \nu(F)+\varepsilon / 2\right\} .
$$

If $q \in V$, choose an open set $U$ such that (i) $F^{q} \subseteq U$, (ii) $X \backslash U$ is compact, and (iii) $\mu(U)<\mu \times_{2} \nu(F)+\varepsilon / 2$. By Lemma 4.1, $F^{y} \subseteq U$ for all $y$ in some neighborhood of $q$. Thus $V$ is open. Since $B \subseteq V$, we see that $\nu(V)=1$. Let $\kappa$ be the smallest cardinal such that there exist $\kappa$ Borel subsets of $V$ of measure 1 whose intersection has zero $\nu$-inner measure. Let $D$ be a subset of $V$ such that the $\nu$-outer measure of $V \backslash D$ is zero and such that $E$ has an accumulation point in $V$ whenever $E$ is a subset of $D$ with cardinality $\kappa$. 
For each $\alpha<\kappa$, we shall find a point $y(\alpha)$ in $B \cap D$ and a Borel set $B_{\alpha}$ contained in $B$ with the following properties:

(i) $y(\alpha) \in B_{\beta}$ and $B_{\alpha} \subseteq B_{\beta}$ for all $\beta<\alpha$,

(ii) $\nu\left(B_{\alpha}\right)=1$, and

(iii) $\mu\left(F^{y} \triangle F^{y(\alpha)}\right) \geqslant \varepsilon$ for all $y$ in $B_{\alpha}$.

Assuming that such $y(\beta)$ and $B_{\beta}$ have been found for all $\beta<\alpha$, let $G_{\alpha}=\cap\left\{B_{\beta}\right.$ : $\beta<\alpha\} \cap B$. Choose a Borel set $C_{\alpha}$ contained in $G_{\alpha}$ such that $\nu\left(C_{\alpha}\right)=1$. Since $D$ meets every Borel set in $V$ of positive $\nu$-measure, the set $C_{\alpha} \cap D$ is nonempty. Choose $y(\alpha) \in C_{\alpha} \cap D$. For $N=F^{y(\alpha)} \times Y$, let $B_{\alpha}=\left\{y \in Y: \mu\left((F \triangle N)^{y}\right)=\right.$ $\left.\mu \times{ }_{2} \nu(F \triangle N)\right\} \cap C_{\alpha}$. Then $B_{\alpha}$ is a Borel set and $\nu\left(B_{\alpha}\right)=1$. If $y \in B_{\alpha}$, then $\mu\left(F^{y} \triangle F^{y(\alpha)}\right)=\mu\left((F \triangle N)^{y}\right) \geqslant \varepsilon$ since $\mu \times_{2} \nu(F \triangle N) \geqslant \varepsilon$ for each $N \in \mathscr{B}(X) \times$ $\mathscr{B}(Y)$. It is thus easy to see that $\{y(\alpha)\}$ and $\left\{B_{\alpha}\right\}$ have the required properties.

If $\beta<\alpha$, then $y(\alpha) \in B_{\beta}$ and $\mu\left(F^{y(\alpha)} \triangle F^{y(\beta)}\right) \geqslant \varepsilon$. Thus the $y(\alpha)$ 's are distinct, and $E=\{y(\alpha): \alpha<\kappa\}$ has an accumulation point $q$ in $V$. Since $\mu\left(F^{q}\right)<$ $\mu \times_{2} \nu(F)+\varepsilon / 2$, there exists a set $U$ in $X$ such that (i) $U$ contains $F^{q}$, (ii) $X \backslash U$ is compact, and (iii) $\mu(U)<\mu \times_{2} \nu(F)+\varepsilon / 2$. By Lemma 4.1, $F^{y}$ is a subset of $U$ for all $y$ in some neighborhood of $q$. Let $y(\alpha)$ and $y(\beta)$ be distinct members of $E$ lying in that neighborhood. Then $F^{y(\alpha)}$ and $F^{y(\beta)}$ are both contained in $U$. Since

$$
\mu\left(F^{y(\alpha)} \backslash F^{y(\beta)}\right) \leqslant \mu\left(U \backslash F^{y(\beta)}\right)=\mu(U)-\mu\left(F^{y(\beta)}\right)<\varepsilon / 2
$$

and similarly since $\mu\left(F^{y(\beta)} \backslash F^{y(\alpha)}\right)<\varepsilon / 2$, we see that $\mu\left(F^{y(\alpha)} \triangle F^{y(\beta)}\right)<\varepsilon$ in contrast to the earlier statement that $\mu\left(F^{y(\alpha)} \triangle F^{y(\beta)}\right) \geqslant \varepsilon$. Hence, $\mu \times_{2} \nu$ is well behaved.

COROLlary 4.5. Suppose $\mu$ is a regular Borel measure, and suppose $\nu$ is a purely atomic Borel measure such that every open set of $Y$ is $\nu$-concentrated. Then each closed set in $X \times Y$ contains a closed set in $\mathscr{B}(X) \times \mathscr{B}(Y)$ with arbitrarily close $\mu \times{ }_{2} \nu$-measure. Hence, each closed set in $X \times Y$ contains a set in $\mathscr{B}(X) \times \mathscr{B}(Y)$ with the same $\mu \times_{2} \nu$-measure.

The regularity of $\mu$ is needed in Corollary 4.5. For if $\mu$ is Dieudonne's nonregular Borel measure on $X=\omega_{1}+1[6$, Exercise 52.10] and if $F=\{(x, y) \in X \times X$ : $x \geqslant y\}$, then $F$ is a closed set in $X \times X$ such that $\mu \times_{2} \mu(F)>\mu \times_{1} \mu(F)$ (cf. [7, §6]).

Similarly, the hypothesis that $\nu$ be purely atomic cannot be dropped in Corollary 4.5. For there exist a regular Borel measure $\mu$ on a compact space $X$ and a closed set $F$ in $X \times X$ such that $F$ does not contain a set in $\mathscr{B}(X) \times \mathscr{B}(X)$ with the same $\mu \times_{2} \mu$-measure [3].

A set will be called nearly closed if it contains the closure of each of its separable subsets; the nearly Borel sets are the $\sigma$-algebra generated by the nearly closed sets. If $\mu$ is a regular Borel measure on $X$, then $\mu$ can be extended to a measure $\mu^{\prime}$ on the nearly Borel sets of $X$ [9, Theorem 2.6]. In addition, if $\nu$ is purely atomic, then $\mu^{\prime} \times{ }_{1} \nu(M)$ is defined for all $M \in \mathscr{B}(X \times Y)$ [9, Theorem 3.3]. 
CoROllary 4.6. Suppose $\mu$ is a regular Borel measure on $X$, and suppose $\nu$ is a purely atomic Borel measure on $Y$ such that every open set in $Y$ is $\nu$-concentrated. Then $\mu \times_{2} \nu(F) \leqslant \mu^{\prime} \times_{1} \nu(F)$ for each closed set $F$ in $X \times Y$ and each extension of $\mu$ to a measure $\mu^{\prime}$ on the nearly Borel sets of $X$.

Theorem 2.1 and Corollary 4.5 can be combined to give conditions under which $\mu \times_{1} \nu$ and $\mu \times_{2} \nu$ agree for all Borel sets in $X \times Y$.

THEOREM 4.7. Suppose $\mu$ is a regular, separable-regular Borel measure, and suppose $\nu$ is a purely atomic Borel measure such that every open set of $Y$ is $\nu$-concentrated. If $F$ is a closed set in $X \times Y$ such that $\mu \times_{1} \nu(F)$ is defined, then $\mu \times_{1} \nu(F)=$ $\mu \times_{2} \nu(F)$. Hence, if $\mu \times_{1} \nu(M)$ is defined for all Borel sets $M$ in $X \times Y$, then $\mu \times_{1} \nu(M)=\mu \times_{2} \nu(M)$ for all Borel sets $M$ in $X \times Y$.

5. Examples. Often if something "goes wrong" for finite Borel measures in Hausdorff spaces, the same behavior already exists for finite Borel measures in compact spaces. Accordingly, the examples of this section will be given for compact spaces even though an extra step may sometimes be needed to make sure that the spaces are compact.

Our first example shows that we need the hypothesis that $\mu$ be regular in Theorem 1.2. Indeed, we have $\mu \times_{1} \nu(W)>\mu \times_{2} \nu(W)$ for some open set $W$ in $X \times Y$ even if $X$ is first countable and $\mu$ is separable-regular.

Example 5.1. Assume the Continuum Hypothesis. Let $X$ be a first countable, hereditarily separable, compact space possessing a nonatomic Borel measure $\mu$ such that $\mu\left(X_{0}\right)>0$ for some open, locally cou. table subset $X_{0}$ in $X$. Juhász, Kunen and Rudin have constructed such a space under the Continuum Hypothesis [11, p. 1001]. Let $Y$ be $\omega_{1}+1$ with the order topology, and let $\nu$ be Dieudonné's nonregular two-valued Borel measure on $Y$.

By the Continuum Hypothesis, there exists a one-to-one mapping from $X_{0}$ into $\omega_{1}$, say $g: X_{0} \rightarrow \omega_{1}$. For each $x \in X_{0}$, let $U(x)$ be a countable open neighborhood of $x$ and let $V(x)=\left\{y \in Y: g(x)<y<\omega_{1}\right\}$. If

$$
W=\bigcup\left\{U(x) \times V(x): x \in X_{0}\right\},
$$

then $\mu \times_{1} \nu(W)=\mu\left(X_{0}\right)$ and $\mu \times_{2} \nu(W)=0$ since $W^{y}$ is countable for each $y<\omega_{1}$.

Incidentally, if $\mu$ is the measure described in Example 5.1, then $\mu \times_{1} \mu(M)$ and $\mu \times_{2} \mu(M)$ are defined for all Borel sets $M$ in $X \times X$ since $X$ is first countable. Does $\mu \times_{1} \mu(M)=\mu \times_{2} \mu(M)$ for each Borel set $M$ in $X \times X$ ? The author does not know.

In Example 5.1 the domain of completion of $\mu \times \nu$ did not include all the Borel sets of $X \times Y$ for the simple reason that $\mu \times_{1} \nu$ and $\mu \times_{2} \nu$ were not equal. In the next example, $\mu \times_{1} \nu(M)=\mu \times_{2} \nu(M)$ for all Borel sets $M$ in $X \times Y$, but there exists an open set in $X \times Y$ which fails to be $\mu \times \nu$-measurable.

EXAMPLE 5.2. Let $X_{0}$ be an uncountable, locally countable, locally compact space such that each open set in $X_{0}$ is countable or cocountable, and let $X$ be its 
one-point compactification. Ostaszewski has constructed such a space using the combinatorial principle $\hat{Q}[17$, p. 506]. It is easy to see that $X$ is hereditarily separable and that $\operatorname{card}(X)=\omega_{1}$. Moreover, the Borel sets of $X$ are precisely those sets which are countable or cocountable. Let $\mu$ be that Borel measure on $X$ such that countable sets have measure 0 and cocountable sets have measure 1 . Let $Y=X$, and let $\nu=\mu$.

Since $\mu$ is separable-regular, we know by Theorem 2.1 that $\mu \times_{1} \nu(F) \leqslant$ $\mu \times_{2} \nu(F)$ for each closed set $F$ in $X \times Y$. By interchanging the roles of $\mu$ and $\nu$, we see that $\mu \times_{2} \nu(F) \leqslant \mu \times_{1} \nu(F)$ for each closed set $F$ in $X \times Y$. Hence, $\mu \times_{1} \nu$ and $\mu \times_{2} \nu$ agree on closed sets and thus on all the Borel sets of $X \times Y$.

Now let $W=\{(x, y): x \neq y\}$ Since $X=Y$ is Hausdorff, $W$ is open. Clearly $\mu \times_{1} \nu(W)=1$. We notice that $W$ does not contain a Borel rectangle with the same $\mu \times_{1} \nu$-measure. For if $A \times B$ is a Borel rectangle contained in $W$, then $A$ and $B$ are disjoint and at least one of them has measure zero. Hence, $\mu \times_{1} \nu(A \times B)=0$. Since each set in $\mathscr{B}(X) \times \mathscr{B}(Y)$ contains a Borel rectangle with the same $\mu \times \nu$-measure, we see that $W$ contains no set in $\mathscr{B}(X) \times \mathscr{B}(Y)$ with the same $\mu \times_{1} \nu$-measure.

Our next example provides a reminder that $\mu \times_{1} \nu(F)$ may fail to be defined for some closed set $F$ in $X \times Y$ even though $\mu$ is a regular, separable-regular Borel measure on $X$.

EXAMPLE 5.3. Let $X$ be the space of all functions from $\omega_{1}$ into the discrete space of two elements $\{0,1\}$, and let $X$ have the product topology. (Unlike the Cantor set $Z$ in the proof of Theorem 2.4 , notice that $X$ is not first countable.) Let $\mu$ be Haar measure on $X$. Since each compact set in $X$ contains a compact Baire set with the same Haar measure [6, Theorem 64.H] and since each compact Baire set in $X$ is homeomorphic to the product of a compact metric space with the separable space $X$ [6, Exercise 50.4], it follows that $X$ is separable-regular.

Now let $Y=\omega_{1}+1$, and let $\nu$ be Dieudonné's nonregular Borel measure on $Y$. By Theorem 4.7, $\mu \times_{1} \nu(F)=\mu \times_{2} \nu(F)$ whenever $F$ is a closed set in $X \times Y$ for which $\mu \times_{1} \nu(F)$ is defined. If

$$
F=\left\{(x, y): x_{\alpha}=1 \text { if } \alpha \geqslant y\right\},
$$

then $F$ is a closed set in $X \times Y$ for which $\nu\left(F_{x}\right)$ is neither measurable nor $\mu$-almost everywhere measurable [8, Example 3.2].

Our last example shows that if there exists a measurable cardinal, then there exist a regular Borel measure $\mu$ and a two-valued Borel measure $\nu$ such that $\mu \times_{2} \nu$ is not well behaved with respect to $\mathscr{B}(X) \times \mathscr{B}(Y)$. Hence, the conclusion of Corollary 4.5 fails in this case. Recall that a cardinal $\kappa$ is measurable if there exist a set $Y_{0}$ with cardinality $\kappa$ and a two-valued measure defined on the class of all subsets of $Y_{0}$ such that singleton sets have measure zero [10, A6.1].

EXAMPLE 5.4. Assume the existence of a measurable cardinal $\kappa$. Let $Y_{0}$ be a discrete space with cardinality $\kappa$, and let $Y$ be the one-point compactification of $Y_{0}$. Let $\nu$ be a two-valued measure on the class of all subsets of $Y$ such that singleton sets have measure zero. Let $X$ be the space of all functions from $Y$ into the discrete space of two elements $\{0,1\}$, and let $X$ have the product topology. (Warning. This 
space $X$, if it exists, is a far bigger space than the similar-looking space $X$ of Example 5.3.) Let $\mu$ be Haar measure on $X$.

If $x \in X$ and $y \in Y$, then $x_{y}$ will denote the $y$ th-coordinate of $x$. Let $F=$ $\left\{(x, y): x_{y}=1\right.$ if $\left.y \in Y_{0}\right\}$. It can be seen that $F$ is closed in $X \times Y$ since $\{x$ : $\left.x_{y}=0\right\} \times\{y\}$ is open for all $y$ in $Y_{0}$. If $A \times B$ is a closed rectangle contained in $F$, then $\mu(A)$ or $\nu(B)$ is zero. By Theorem 4.3 , we see that $\mu \times_{2} \nu$ is not well behaved.

\section{REFEREN่CES}

1. S. K. Berberian, Measure and integration, Macmillan, New York, 1965.

2. S. P. Franklin, Spaces in which sequences suffice, Fund. Math. 57 (1965), 107-115.

3. D. H. Fremlin, Products of Radon measures: a counter-example, Canad. Math. Bull. 19 (1976), 285-289.

4. R. J. Gardner, The regularity of Borel measures and Borel measure-compactness, Proc. London Math. Soc. (3) 30 (1975), 95-113.

5. S. L. Gulden, W. M. Fleischman and J. H. Weston, Linearly ordered topological spaces, Proc. Amer. Math. Soc. 24 (1970), 197-203.

6. P. R. Halmos, Measure theory, Van Nostrand, New York, 1950.

7. R. A. Johnson, On product measures and Fubini's theorem in locally compact spaces, Trans. Amer. Math. Soc. 123 (1966), 112-129.

8. 28 (1979), 346-352. , Measurability of cross section measure of a product Borel set, J. Austral. Math. Soc. Ser. A

9. $ـ$ Nearly Borel sets and product measures, Pacific J. Math. 87 (1980), 97-109.

10. I. Juhász, Cardinal functions in topology, Math. Centre Tracts, no. 34, Mathematisch Centrum, Amsterdam, 1971.

11. I. Juhász, K. Kunen and M. E. Rudin, Two more hereditarily separable non-Lindelöf spaces, Canad. J. Math. 28 (1976), 998-1005.

12. J. Keesling, Normality and properties related to compactness in hyperspaces, Proc. Amer. Math. Soc. 24 (1970), 760-766.

13. J. L. Kelley, General topology, Van Nostrand, New York, 1955.

14. K. Kunen, $A$ compact L-space under $C H$, Technical Report \#21, University of Texas at Austin, 1980.

15. E. Marczewski and R. Sikorski, Measures on nonseparable metric spaces, Colloq. Math. 1 (1948), $133-139$.

16. D. A. Martin and R. M. Solovay, Internal Cohen extensions, Ann. Math. Logic 2 (1970), 143-178.

17. A. J. Ostaszewski, On countably compact, perfectly normal spaces, J. London Math. Soc. 14 (1976), $505-516$.

Department of Mathematics, Washington State University, Pullman, Washington 99164 